\title{
Alternatives in the Implementation of Internet-Enabled Laboratory Experiments in Undergraduate Civil Engineering Courses
}

\author{
Abhinav Gupta, Mohammed A. Gabr, and Vernon C. Matzen \\ Assistant Professor of Civil Engineering, North Carolina State Univer sity/ \\ Professor of Civil Engineering, North Carolina State Univer sity/ Professor of \\ Civil Engineering and Director, Center for Nuclear Power Plant Structures, \\ Equipment and Piping, North Carolina State University
}

\begin{abstract}
Recent developments in information technology have generated considerable interest in the field of internet-enabled remote observation and control of laboratory experiments. This paper describes two alternative frameworks required to adapt two laboratory experiments, one each in the areas of structural and geotechnical engineering, for remote control and observation including protection from misuse. Faculty and students will be able to observe, conduct, control, and analyze experimental data either in or outside of a classroom. In the face of continuously evolving distance education programs, more and more educators recognize the importance of a complete educational experience involving theory as well as hands-on experiments. In general, a large number of students lack opportunities to gain hands-on experiences due to the availability of only a few experimental facilities nationwide. Opportunities can be created by the availability of such facilities via remote access and control within a distance learning environment. Even for on-campus students, the possibility of experimental training can be unrealized due to economic constraints in developing a large number of experimental set-ups for simultaneous use in a laboratory environment within a limited allotted time. Simulations (virtual experiment) are often used to integrate classroom lecture with some kind of hands-on experience. However, educators have pointed out that simulation systems, while important, cannot completely substitute for experience with actual materials. One of the two implementations considered in this paper uses LABVIEW for not only data acquisition and control but also for providing web access and for developing features to safeguard against the possibility of damage to the experimental setup if left unmonitored. The other implementation considers the use of equipment-specific software for control in conjunction with MS-Netmeeting for web accessibility. The advantages and limitations associated with each implementation are discussed with respect to the experiments being adapted. Different options available within LABVIEW are also presented and our experiences on the advantages and limitations of these options discussed.
\end{abstract}

Introduction

Comprehension of concepts in engineering mechanics and materials is a challenging task for undergraduate students. In a conventional classroom setting, students often find it difficult to

"Proceedings of the 2004 American Society for Engineering Education Annual Conference \& Exposition Copyright (C) 2004, American Society for Engineering Education" 
visualize the mechanistic behavior of, for example, beam and column deformations, timedependent soil deformation, or changes in the shear strength of soil under drained and undrained conditions. While our curriculum consists of several laboratory experiences, it is impractical in a majority of cases to supplement the in-class lectures with experimental demonstration illustrating important concepts such as time-dependent deformation, multiple failure modes, and dependency of measured parameters on loading rates. The capability to integrate experimental demonstrations with classroom lectures can re-energize the conventionally taught courses. Student learning could be improved by actively engaging them in observing, conducting, controlling, and analyzing experimental data for discerning material behavior while understanding uncertainties through experimental measurements and demonstration. Additionally, students in rural communities where hands-on laboratory experience is not available could have access to laboratory equipment where they can remotely conduct and control experiments.

\section{Overview of Recent Work}

Developments in information technology over the last decade have produced a considerable amount of activity in the field of internet-enabled experiments. Significant emphasis has been placed on the development of internet-enabled remote collaboratories under the NSF sponsored National Earthquake Engineering Simulator (NEES) program. In the face of continuously evolving distance education programs, more and more educators recognize the importance of a complete educational experience involving theory as well as hands-on experiments ${ }^{1}$. Even for on-campus students, the possibility of experimental training can be unrealized due to economic constraints in developing a large number of experimental set-ups for simultaneous use in a laboratory environment. Simulations (virtual experiment) are often used to integrate classroom lecture for some kind of laboratory experience ${ }^{2}$ such as a multimedia interactive teaching module that has been developed to illustrate basic concepts of one-dimensional consolidation of soils ${ }^{3}$. However, researchers have pointed out that "There will always be an important place of simulation systems, but they cannot completely substitute for experience with actual systems ${ }^{4} . "$ For instance, simulations do not provide any insight into calibration of measuring instruments such as pressure gages and LVDT's or into behavioral uncertainties. As discussed by some researchers ${ }^{1}$, the conceptual idea proposed herein can provide remote laboratory users the opportunity to conduct live experiments off-site thereby reducing the experiment cost per student and making experiments available to many more users. Key issues are: (a) use of the internet as a communication infrastructure between the student and the equipment, which may be in geographically different locations, and (b) the adaptation of these experiments to be remotely controlled.

Several researchers in the area of control engineering have successfully developed remotely controlled experimental setups for both the in-class teaching and distance learning ${ }^{5-7}$. A webbased tutorial and tele-operation system for earthquake engineering education has been developed at Southern Illinois University ${ }^{8}$. However, it did not address remote control. Once the input values were set, remote viewing and analysis were the only objectives. Changes to the inputs were not needed. "The Web pages allowed students and interested engineers to remotely access the website and learn by watching the $3 \mathrm{D}$ graphics model ${ }^{8}$." As another example, a distance learning application that did include remote control of laboratory experiments has been developed at Oregon State University ${ }^{9}$.

"Proceedings of the 2004 American Society for Engineering Education Annual Conference \& Exposition Copyright (C) 2004, American Society for Engineering Education” 
As outlined by researchers in the NEES program, adaptation and implementation of these preliminary studies in the development of Civil Engineering related research collaboratories has been limited due to practical difficulties that relate to user-friendly technology, logistics, copyright, safety, security, and cost associated with research related large-scale testing (www.neesgrid.org). Their success dependencies are outlined as:

- Productive communications environment for collaboration

- Acquisition, management and visualization of massive amounts of digital, video, photo, and numeric data

- Reliable distribution of real-time data

- Curation, synthesis, analysis, management, mining, dissemination and findings from various experiments and comparisons.

The research outlined in this paper has the same initial three goals but places the highest importance on developing education abilities.

\section{Educational Value}

Converting our conventional teaching laboratories into a distance-learning lab will result in significantly increased educational value. Not only will the quality of learning for present students be increased by this change, but also the number of students reached, and the diversity of this group, will be enhanced. Through distance learning, non-traditional students such as underrepresented minorities, working parents, part-time students, students with disabilities, and career changing adults could all benefit from the exercises.

Learning and retention of lecture material can be improved through allowing active participation by the students. The use of observation, control, and analysis of experiments are each beneficial towards increased learning and can be used in conjunction with or as an alternative to passive listening and reading exercises. In fact, some studies have shown that students do more critical thinking in their online exchanges than in face-to-face classroom discussion ${ }^{10}$. By using a distance learning laboratory students will gain hands on experience while the need for an instructor is optional. For example, they will have the ability to actually control a shake table in one of our applications giving them hands on knowledge of vibration experimentation.

This type of implementation holds additional appeal for non-traditional students. By placing courses online, students no longer need to commute to a campus. This can save both time and monetary resources, thus opening the door for those who otherwise would not be able to gain further education. Those with physical disabilities would benefit from the same factors. Lessons and experiments could be accessed from their own homes.

The development of collaboratories mentioned earlier also has educational value. The use of a remotely operated structures lab would stimulate the development of shared resources between other institutions and colleges. Students at community colleges and smaller schools would have an opportunity to use equipment previously inaccessible to them.

Geotechnical Engineering Experiment

In many institutions across the country and worldwide, soil mechanics laboratory is a mainstay of undergraduate Civil, Environmental, and Construction Engineering curricula. Given the inherent variability of soils, laboratory experience gives students an appreciation of the judgment 
required in geotechnical analysis and design as well as the uncertain nature of the measured properties. Observing soil behavior under compression and shear helps students understand challenging concepts, such as the fact that these properties are time and load dependent.

One of the tests conducted in soil mechanics laboratories is Consolidated Undrained Triaxial Compression Test for Cohesive Soils (ASTM D4767-95). This test is used for estimating the strength and stress-strain relationship of a cylindrical soil specimen subjected to confining stress and sheared in compression without drainage at a constant rate of axial deformation (strain controlled). The specimen size varies but typically is $50 \mathrm{~mm}$ in diameter and $150 \mathrm{~mm}$ in height. In general, three specimens are tested at different effective stress levels to define a failure envelope. In order to automate this test, the axial stress is applied using low torque motor control with a screw actuator that transfers the load to the sample using a piston. An electronic load cell is used to measure the axial load. The axial deformation is monitored using an LVDT and the pore pressure within the sample is monitored using pore pressure transducers. The chamber pressure is controlled and monitored using digital pneumatic pressure regulators that are capable of applying and controlling pressures within $+/-2 \mathrm{kPa}$.

Implementation Using Equipment-specific Control Software: For equipment that has its own control software, such as the geotechnical engineering experiment considered in this study, the simplest way to remotely operate it is to use equipment-specific software in conjunction with control software such as Microsoft Net-meeting. Net-meeting permits password protected access of a particular computer to the users and allows them to run the experiment software remotely. A version of Microsoft Netmeeting ${ }^{\mathrm{TM}}$ is used to remotely run the experiment and control the equipment computer. The test chamber for the triaxial experiment is shown in Fig. 1 (a) while the computer-controlled loading frame is shown in Fig. 1(b).
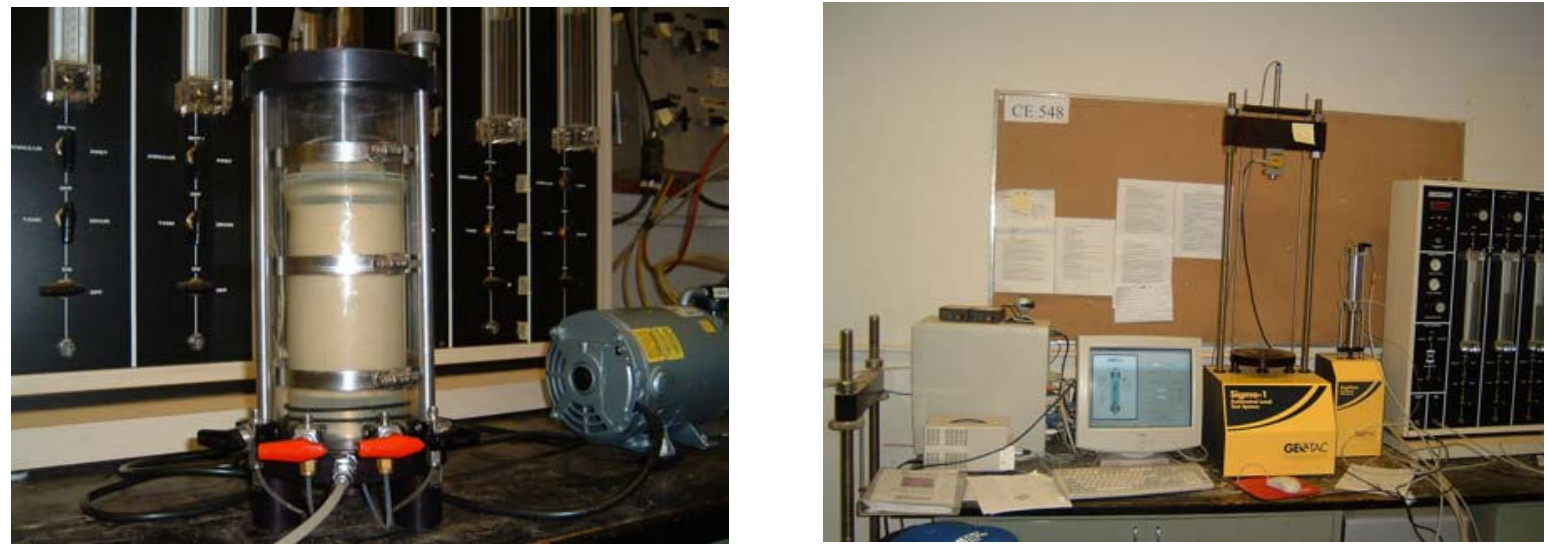

Figure 1. Set for Triaxial Experiment: a) Testing Chamber and b) Loading Frame 
Software Setup for Triaxial Experiment

The software needed to remotely control the experiment and collect the data include three programs as follows:

Microsoft Netmeeting $^{\mathrm{TM}}$ : Most Windows ${ }^{\mathrm{TM}}$ operating systems come with a version of Microsoft Netmeeting ${ }^{\mathrm{TM}}$. This program enables users to remotely access other computers. Setting up Netmeeting is easy as one only double clicks on the Netmeeting icon to open the program. Next, one enters in the IP address of the computer being contacted. Finally, the "telephone" button on the right side of the window is activated to access the caller. The computer receiving the call must be set to receive "phone" calls.

Digiflow-GP: This program runs the pump that controls and monitors the water pressure. This pressure was preset to 10,20 , or $40 \mathrm{psi}$ as specified for the experiment. Once the correct pressure level is set, the pump is activated. The pressure can be monitored in a "pressure" window. Before testing has begun, the pump may need to be adjusted to allow it to reach the desired pressure.

Sigma 1-CU: Essentially, this program controls the test and collects the data. Other than the constant pressure controlled by the pump along with the Digiflow-GP software, this program runs the entire test and records all pressure and deformation data. This software controls the motor equipment which applies the load to the soil sample until failure. The strain rate and reading schedules can be modified to a desired resolution so that the user can maximize data collection at important points, as well as ensure that testing is conducted for the desired period of time. The displacement for a sample is calculated as a function of its measured height and with time. As a part of this research, an undergraduate student was given the task of running the experiment remotely and then in the lab in order to provide comments on challenges he faced. Fig. 2 shows stress-strain data obtained from the same test run remotely and then in the

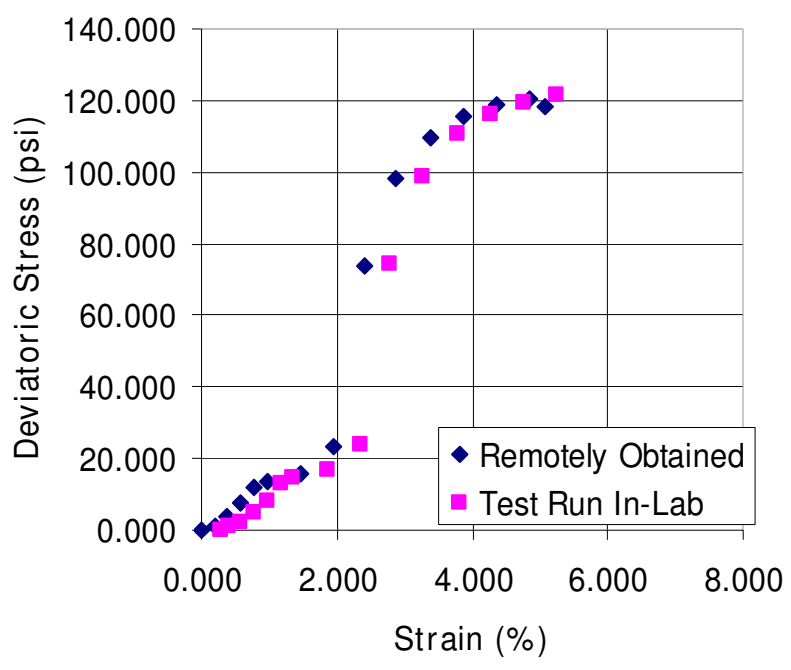

Figure 2. Results Obtained from Test Run Remotely Versus Test Run in Lab Challenges with Remote Triaxial Experiment (40 psi). 
laboratory. Both tests which were conducted by one undergraduate student considered same soil (sand) and the same confining pressure. Both tests were run by an undergraduate student. The results are virtually identical within the limits of experimental errors and the inherit variability in sample properties due to variation induced during preparation.

The student summarized the remote experience with the following statement "The testing in general was of good quality and full of frustrating moments." A summary of challenges faced are:

i. Test Set up: it was a challenge learning how to set up the test sample properly. Once the O-rings and membrane were attached to the base of the tri-axial device, sand was added at a specifically desired density. Attaching the cylinder wall and the top of the chamber had to be done very delicately. The sample set up time was approximately 20 minutes. These challenges are however inherent in this type of testing whether or not it is performed remotely.

ii. During Consolidation Phase: applying confining pressure to the sample went flawlessly, except in a few instances where the sample leaked under relatively high pressure. Using $40 \mathrm{psi}$, the sample sprang a small leak at the bottom of the cylinder and caused the test to be a loss. In this instance the sample had to be taken completely apart, equipment cleaned, and reconfigured.

iii. During the Shearing Phase: testing went almost flawlessly. These tests were run under 10,20 , and 40 psi confining stress. The only issue that arose when dialing the computer in the laboratory using Microsoft Netmeeting ${ }^{\mathrm{TM}}$ is getting error message stating: "The other party did not accept your call." This issue was resolved by simply clicking on "call" and then selecting "automatically accept calls" for the host computer.

\section{Shake Table Experiment}

The shake table considered in this study is used primarily for teaching purposes at the undergraduate and graduate levels. It is a 12" x 34" one-dimensional table with a $50 \mathrm{lb}$ electromagnetic shaker. In the undergraduate curriculum, Junior-level students use this experiment as part of a 1 credit-hour laboratory course on structural behavior measurements. The particular experiment focuses on evaluating the natural frequency and damping ratio for a single story shear building having wide but thin Aluminum columns and a heavy steel girder. A schematic diagram of this setup is shown in Fig. 3. The forced vibration test is conducted by applying a harmonic excitation to the table and then measuring the acceleration response of the table and the girder. The frequency of excitation is varied in steps from a value that is much lower than the natural frequency of the shear building to one that is much higher. For each input frequency, the table is excited for a duration that is sufficiently long to guarantee steady state motion. The ratio of the girder's acceleration amplitude to table's acceleration amplitude is then used to evaluate the dynamic magnification factor for the particular frequency. The dynamic magnification factors calculated for a range of frequencies are then used to evaluate the natural frequency and damping ratio of the shear building in accordance with the formulations given in undergraduate dynamics textbooks.

It should be noted that the shear building models used in the experiments mentioned above are constructed using thin aluminum columns. These columns are susceptible to fatigue 
failure which can occur rather quickly if the shear building is excited either for long durations at low amplitude or for short duration at high amplitudes, e.g. in the resonance or near-resonance regions. Depending upon the type of setup, the shake table and the actuator may also be prone to damage if excited at very high amplitudes. Thus, several safety issues must be considered when remotely accessing and controlling the shake table. Some of these safeguards are needed for protection against misuse whereas others are needed to safely shutdown the experiment if the electronic communication between the experimental hardware and the controlling computer is disrupted.

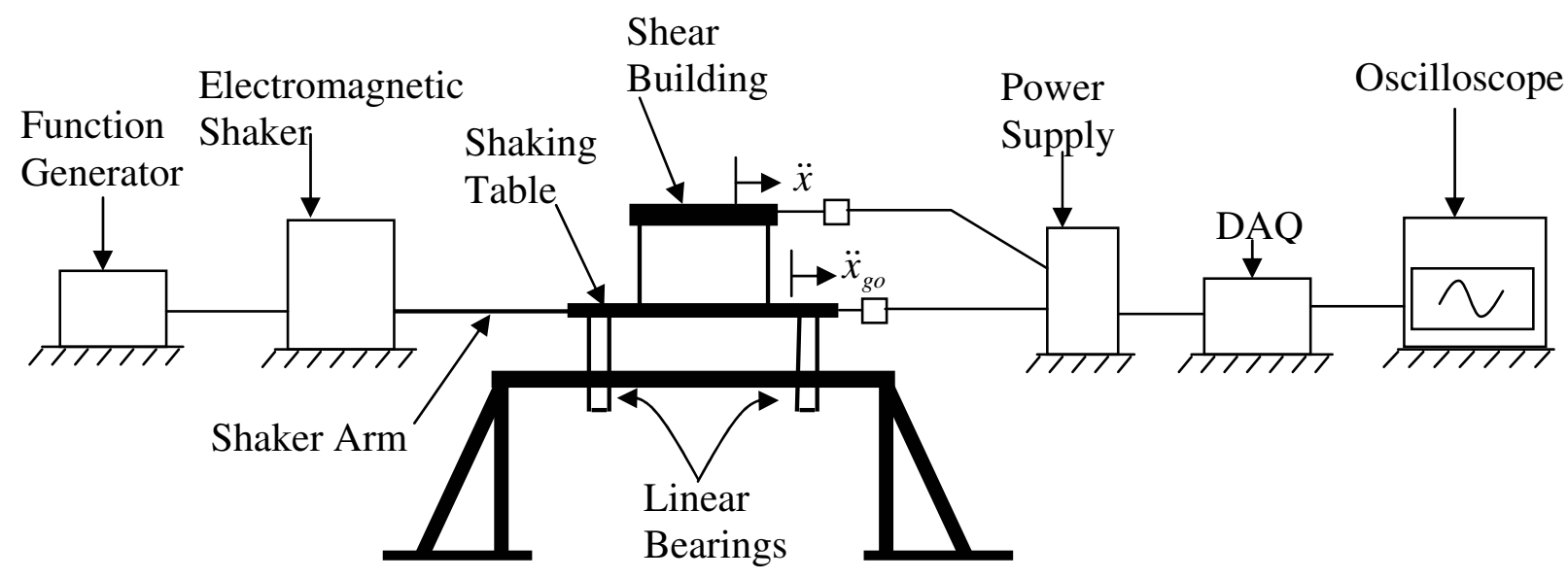

Figure 3 - Shake table setup

Implementation Using LabVIEW for Control and Web-based Access: Unlike the geotechnical engineering experiment, forced vibration experiment with shake-table does not require human intervention. The rate of loading is relatively fast, and the test equipment does not have its own controller. Because of this last reason, we could consider the use of other control software and relinquish the dependence on Microsoft Netmeeting. Since we have used LabVIEW software by N.I. ${ }^{12,13}$ in our lab for many years, we chose it to remotely access and control the shake-table experiment considered in this study. LabVIEW has built-in capabilities for internet-enabled instrumentation that include TCP/IP and UDP functions, built-in web server to create front panel images, VI server for network-based communication, etc. LabVIEW can also be used with other technologies and software such as Java applet for remote control and observation, ActiveX controls, CGI support, E-mail, etc. The graphical panels developed within LabVIEW for user interfaces are referred to as front panels, whereas the complete block diagram based code is referred to as a Virtual Instrument, VI.

In its simplest form, a LabVIEW based setup would consist of an administrator controlled Windows based host computer that uses LabVIEW to communicate directly with the DAQ. Such an implementation would require two VI's within LabVIEW, one for controlling the table by generating and sending the input waveform and the other for performing oscilloscope functions to display and collect the acceleration data. In an implementation of this type, however, a disruption of electronic communication between the host-PC and the DAQ, such as that due to the failure of the operating system, can leave the experiment unmonitored and susceptible to damage. Safeguards against such situations require high-performance real-time data acquisition and control which is difficult to attain using off-the-shelf computer technologies. As discussed in 
detail in the next section, we accomplish this by using separate hardware and software for realtime control. The host-PC can also be used directly to provide web access to the remote user. The network security can be enhanced by using a proxy server together with the host computer within a client-server environment.

LabVIEW Implementation Developed for Real-time Control of a Shake Table Experiment

In its simplest form, one may consider executing the control software for conducting the experiment directly on the host-PC. However, the performance of such an implementation would be dependent upon the hardware characteristics of the host-PC thereby making it highly unreliable. It is critical that the real-time control software and the operating system such as Windows be assigned independent processors and memories that are dedicated to each task in order to achieve a reliable implementation for safe shut down. Otherwise, unavailability of resources on a single processor and shared memory due to an operating system crash will prevent the real-time software from executing a safe shutdown. Even under normal operating conditions, the various operating system processes can reduce the feedback time for controlling the shake table when a single processor and shared memory are used for executing both the tasks. Standardized hardware containing real-time control boards with a dedicated processor and independent memory are available for implementing such a solution. Such a board provides the capability of embedding LabVIEW programs into them for real-time (RT) control ${ }^{13}$. Clearly, communication with DAQ must then be performed through the real-time board. In our hardware setup the DAQ controller is located on the same card, i.e., the same processor is used for realtime control and DAQ control. Fig. 4 shows a schematic of the modified lab setup with real-time control. Since the host-PC and the real-time board can have individual IP addresses, a clientserver environment can be used for communication between the two processors. This arrangement eliminates the dependency of control devices on the execution of the operating system, i.e. an operating system crash on the host-PC will not affect a safe shutdown of the system.

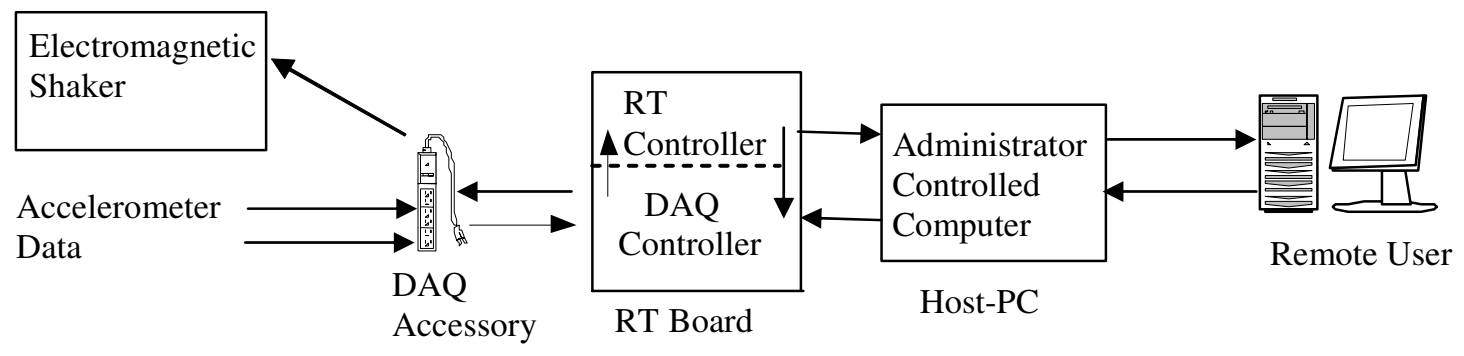

Figure 4 - Experimental setup with real-time hardware

Various safeguard features are implemented by programming the real-time applications using LabVIEW-RT, the real-time counterpart of LabVIEW. For example, LabVIEW RT has built in functions that facilitate communication between programs executing on the host-PC's operating system (such as Windows) and the real-time board. These functions allow the user interfaces for access, control, observation, and data collection to be implemented using the LabVIEW codes located on the host-PC whereas the codes for actual operation and control of the table are implemented in LabVIEW-RT located on the real-time board. It is important to note 
that such an implementation requires a careful handling of various tasks in a particular code for efficiently managing the response and feedback times which can be influenced by the time needed for communication between the LabVIEW and LabVIEW-RT. This framework also requires the development and implementation of another VI for monitoring the progress of successful communication between the two processors and for ordering a safe shutdown when the communication breaks down due to reasons such as an operating system crash. We developed three distinct VIs, i.e. LabVIEW and LabVIEW-RT programs, for implementing the framework discussed above. Specifically, these programs are as follows: Waveform Generation $V I$ to control the table by generating and sending the input wave form; Data Display and Storage $V I$ to perform oscilloscope functions; and Handshaking VI to monitor communication between LabVIEW and LabVIEW-RT and ensure a safe shutdown. The former two VI's are specific to the shake table experiment and are described elsewhere ${ }^{14}$. The Handshaking VI is generic in the sense that it would be needed to extend the proposed framework to any other experiment.

Handshaking VI: As shown in Fig. 5, this VI consists of a sub-VI on the host-PC that continually sends a signal to the real-time board. The corresponding sub-VI on the real-time board listens for this signal. If the signal is received, it continues monitoring. If the stop signal is received, the code exits and closes the TCP/IP connection. Any other programs running on the real-time board are allowed to continue execution. The primary purpose of this feature is to stop the communication between the host-PC and the real-time board and if needed, to restart or reconfigure the host-PC without having to restart the real-time board. The real-time board would not receive any signal if a disruption of electronic communication occurs. If no valid signal is transferred from the host-PC, the code stops execution of every LabVIEW-RT program running on the real-time board. It is important to note that this code is only checking for a proper connection between the PC and real-time processors. Therefore, there is no dependence on the VI's handling the shake table operation and display. The intended purpose is to allow the handshaking code to be modular in nature and execute with other codes using both the PC and real-time processors. The feature that allows the program to be stopped, without shutting down each of the LabVIEW-RT programs executing on the real-time board, provides an added flexibility to the administrator for manually switching off each program that is executing on the real-time board without having to restart LabVIEW-RT.

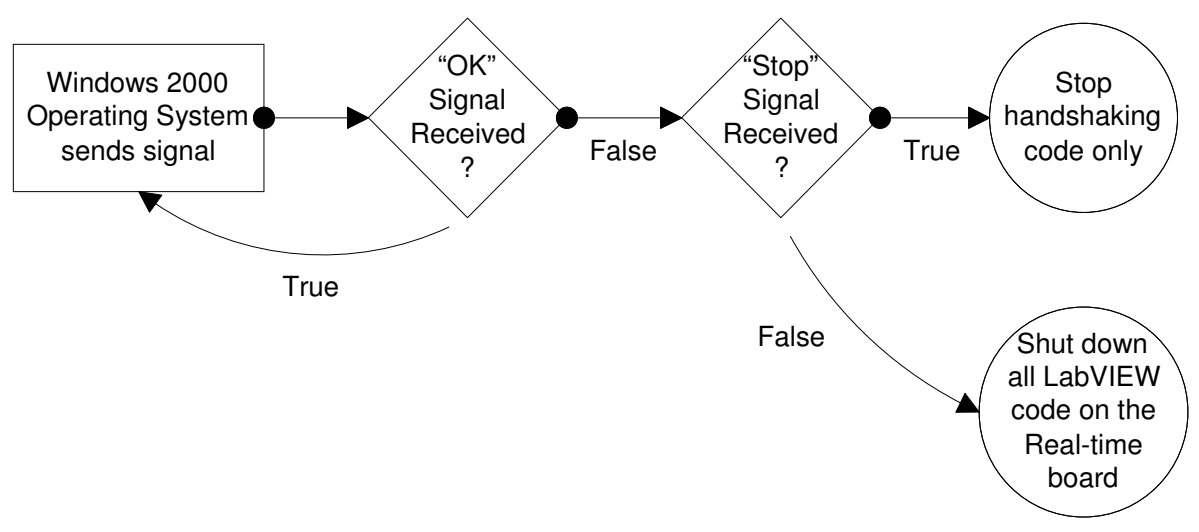

Figure 5 - Flowchart for functionality of handshaking VI

\footnotetext{
"Proceedings of the 2004 American Society for Engineering Education Annual Conference \& Exposition Copyright
} (C) 2004, American Society for Engineering Education" 
Several types of errors are checked and handled by each of the three VIs developed. These include: (a) errors created by improper hard coding for the hardware used, (b) software related errors, and (c) errors due to violation of safeguards against misuse. Note that each element in the error handling is critical for ensuring a safe shut down of the experiment and avoiding any software or hardware related damage. Details of the error handling codes are described elsewhere ${ }^{14}$.

Web Accessibility and Remote Observation: It is important to note that the particular scheme described above and implemented on the host-PC is targeted towards simplifying the implementation for remote access, observation, and control. Web accessibility requires that each VI that is available to the remote users must also reside on the web server. Web hosting can be achieved either directly through the host-PC or by using an additional server. Use of an additional machine is more desirable due to increased safety and reduced load on the host-PC. However, it requires additional resources and may also face issues related to the use of LabVIEW specific plug-ins as discussed below. To facilitate the objective of this initial exploratory work and to maintain the simplicity of implementation, we used the host-PC as the web server. Consequently, no additional work is needed in order to make the two VIs, one for the waveform generation and the other for data display and storage, available to the remote user for I/O. LabVIEW has a built-in web server that was used in this implementation. It allows remote access to a static list of valid IP addresses that is specified by the administrator. It also allows specific IP addresses to be completely blocked and allows different types of access to different IP addresses, i.e. access for only remote observation or access for control in addition to observation.

\section{Alternative LabVIEW Implementations: Parallel Loops and Remote Front Panels}

Next, we would like to discuss two additional implementations that may be viewed as alternatives to the proposed scheme. These are referred to as parallel loops and remote front panels. Parallel loop is a programming structure available within LabVIEW that can be used to reduce the number of VIs. Each of the three primary VI which have been developed in this study is implemented as a pair, i.e. as a VI on the host-PC and its counterpart on the real-time board. All together, they represent three pairs (six individual LabVIEW programs) that can be condensed into a single pair by pursuing parallel loop structure. This pair would consist of only one VI for conducting all the LabVIEW operations on the host-PC and the other VI for conducting them on the real-time board. Such an implementation would improve the startup and shutdown procedures. Also, only a single VI would need to be configured as a visible-VI for remote access, observation, and control. However, the modularity of the proposed implementation will be lost by using a parallel loop structure. Loss of modularity will severely restrict the portability of this implementation to other experiments in future. Modularity also facilitates the implementation of the error handling procedure. Further, it is likely that a single loop within a parallel loop structure would consume most of the available resources on a device thereby slowing down the feedback and even jeopardizing the safe shutdowns.

The use of remote front panels is another LabVIEW option that would eliminate the need of running separate VIs on the host-PC and the related data communication with the real-time board over TCP/IP. In this option, all the VIs can be implemented directly on the real-time board and served to the PC as remote front panels. The PC would then simply provide web-hosting 
services. However, this implementation would require not only a capability for web-serving built into the real-time board but also a hard drive for data storage. These requirements make this option cost-intensive. Thus, we did not consider this option and cannot comment on additional issues related to appropriate error handling and safe shutdowns.

Conclusions:

This paper describes alternative implementations for converting two different laboratory experiments to enable remote access, observation, control, and data collection. In the case of the geotechnical engineering experiment, equipment-specific control software was used and the experiment requires human intervention for sample preparation. Based on the results of the limited study reported in the paper, the following conclusion are advanced:

- A client-server environment accessed using Netmeeting ${ }^{\text {TM }}$ and utilizing off-the-shelf software for controlling the experiment proved to be simple and effective in conducting triaxial soil testing.

- Such a system seems to be flexible enough to extend to other Geotechnical experiments that are already automated and available in the market (such as consolidation and direct shear test.)

- Multiple users may view the experiment being performed and share the data, but only one person is able to control the experiment variables and parameters.

- In case of unexpected "client computer" shut down, the host computer continues to collect data as originally planned by the user. While this is a desirable attribute, it may also pose a safety hazard if control is not re-gained quickly. On the other hand, if the host computer is unexpectedly shut down, the experiment may have to be physically reset.

- Security and safety constraints need to be worked out to prevent unintentional or intentional overloading of the equipment or exceedance of equipment performance constraints.

Key conclusions of the LabVIEW based implementation for the shake-table experiment are:

- A client-server environment with data communication over TCP/IP is the best-suited alternative for maintaining modularity as well as for safeguarding against possible damage.

- Another alternative that involves the use of parallel loop structure within LabVIEW improves the startup and shutdown procedures. However, the modularity of the proposed implementation is lost by doing so.

- The modular implementation developed in this study has the flexibility for application to other experiments with only minor modifications.

- An implementation utilizing parallel loop structure can consume most of the available resources on a device. This can slow down the feedback and jeopardize safe shutdowns.

- A separate handshaking module is necessary to check continuity of communication between the real-time board and the host computer. It enables safe shutdowns when electronic communication is disrupted due to system or network failures.

- Another alternative that involves the use of remote front panels in LabVIEW would eliminate the need of running separate VI's on the host-PC and the data communication with real-time board over TCP/ IP. However, this implementation is cost intensive due to additional hardware requirements.

In this study, we did not compare the Netmeeting implementation directly with the LabVIEW implementation. We anticipate investigating this aspect in future studies. 


\section{Acknowledgements}

This material is based upon the work supported by National Science Foundation under grant No. DUE-0310845 and by the Department of Civil Engineering as well as the College of Engineering at North Carolina State University. The authors acknowledge the work by Graduate students Scott Wirgau, Tanya Kunberger, and undergraduate student Joe Dudeck.

\section{Bibliography}

1. Corradini, M. L., Ippoliti, G., Leo, T. and Longhi, S., "An internet based laboratory for control education," in Proceedings of the 40 ${ }^{\text {th }}$ IEEE conference on Decision and Control, Orlando, FL, December 2001.

2. Oliphant, J. and Oliver, A.W., "Computer-aided learning program for teaching effective stress to undergraduates" Geotechnical and Geological Engineering, v17, n2, Kluwer Academic Publishers Dordrecht Netherlands, 1999, pp 85-97.

3. Budhu, M., "Numerical and visualization techniques in geotechnical engineering education," Geotechnical Special Publication, GeoDenver 2000 Conference 'Educational Issues in Geotechncial Engineering', n109, Denver, CO, 2000, pp 39-47.

4. Bohus, C. L., Crowl., A., Aktan, B. and Shor, M. H., "Running control engineering experiments over the internet, in Proceedings of the $13^{\text {th }}$ IFAC World Congress, San Francisco, CA, 1996, paper no. 4c-03.

5. Yorkovich, S. and Passino, K. M., "An intelligent control laboratory course, in Proceedings of the $13^{\text {th }}$ IFAC World Congress, San Francisco, CA, 1996, paper no. 4c-03.

6. Poindexter, S. E. and Heck, B. S., "Using the web in your courses: What can you do? What should you do?," IEEE Constrol System, vol.9, no.1, 1999, pp. 83-92.

7. Ko, C. C., Chen, B. M., Ramakrishnan, V., Chen, R., Hu, S. Y., and Zhuang, Y., "A large scale web-based virtual oscilloscope laboratory experiment," IEEE Sci. Educ. Journal, vol. 9, no. 2, 2000, pp. 69-76.

8. Smith S. R., Husted, C. W., Smith, S. and Cross, B., "A web-based tutorial and tele-operation system for earthquake engineering education," Proceedings of $30^{\text {th }}$ ASEE/IEEE Frontiers in Education Conference, Kansas City, MO, 2000, October 18-21.

9. Shor, M. and Bhandari, A., "Access to an instructional control laboratory experiment through the world wide web,: in Proceedings of 1998 American Control Conference, Philadelphia, PA, 1998, pp. 1319-1325.

10. Newman, D. R., B. Webb, and C. Cochrane. "A content Analysis Method to Measure Critical Thinking in Faceto-face and Computer Supported Group Learning." Interpersonal Computing and Technology, Volume 3, Number 2, pg. 56-77, 1995.

11. ASTM Standards, Volume 4.08, Soil and Rock (I): D420-D5779, 2001, West Conshohocken, PA.

12. Travis, J., "Internet Applications in LabVIEW," Prentice-Hall, 2000.

13. Wells, L., and Travis, J. "LabVIEW For Everyone," Prentice Hall, 1997.

14. Wirgau, S.A., Gupta, A. and Matzen, V.C., "Internet-Enabled Remote Observation and Control of a Shake Table Experiment," Journal of Computing in Civil Engineering, ASCE, 2004, submitted.

ABHINAV GUPTA is an Assistant Professor of Civil Engineering at NC State University. His research and educational activities have been focused on integration of emerging computing (numerical and information) technologies with the engineering knowledge for improved understanding and visualization of structural 
performance.

MOHAMMED GABR is a Professor of Civil Engineering at NC State University. He has received several national recognitions including ASCE Edmund Freidman Professional Recognition award as well as being named WV young civil engineer of the year in 1996. His research program addresses the innovative applications of geosynthetics for environmental soil remediation and soil improvement

VERNON MATZEN is a Professor of Civil Engineering at NC State University, Alumni Distinguished Professor for Undergraduate Teaching, Member of the Academy of Outstanding Teachers, Director of the Center for Nuclear Power Plant Structures, Equipment and Piping and Director of the Structural Behavior Measurements Laboratory. He conducts research in experimental and analytical mechanics, including inverse problems, in civil and nuclear structures. 